\title{
Echoes of chaos
}

Karl Sigmund

Frontiers of Complexity: The Search for Order in a Chaotic World. By Peter Coveney and Roger Highfield. Faber/Fawcett: 1995. Pp. 462. £18.99, \$27.50.

Hidden Order: How Adaptation Builds Complexity. By John H. Holland. AddisonWesley: 1995. Pp. 185. \$24 (hbk); £18.95 (pbk).

IT is the way of fashion to go out of fashion. The new field of complexity seems to experience the same fate as its forerunner, chaos. After a few years of hope and hype, and a remarkable success on the public relations front, it is hitting lean times. A Scientific American suddenly turned sceptical appears to herald the decline by asking bluntly whether complexity is a sham.

No two researchers in the field agree on what defines complexity: but all make use of the same handful of examples: the brain, the Brazilian rainforest, the immune system and New York City. These systems are wonderfully intricate, evolve in time and offer daunting challenges for computer simulations. But can they be subjected to a unified approach? A battle-cry of chaos was that simple rules can lead to complicated behaviour: now it seems that complicated rules can also lead to complicated behaviour. Is that all it needs to keep pop science going?

Following in the wake of several bestsellers on the subject, Frontiers of Complexity and Hidden Order are latecomers and may have to face a recession. On the other hand, both sides of the complexity debate will find them useful for ammunition. The two books are as different as can be. Frontiers of Complexity is science journalism at its best, succinctly covering an impressively wide range of recent results and carefully tracing back their roots (mostly to Alan Turing and to John von Neumann). Hidden Order, by contrast, offers a highly personal manifesto of complexity, in the form of a series of easygoing lectures, by one of the godfathers of the field.

The authors of Frontiers of Complexity (a physicist and a science journalist who formerly collaborated on the well-received Arrow of Time) have produced an impeccably researched, amazingly up-to-date, crisply written and well-illustrated survey of a plethora of scientific topics that have recently grabbed the media's attention (neural networks, test-tube evolution and quantum computers, to name but a few). These mostly happen to be complex systems, but there is little to suggest an underlying unified science. Actually, apart from occasional sallies against misguided reductionism, the authors do not belabour this point. Extensive coverage is their aim. They have obtained interviews with all the right people, checked and double-checked their information and come up with more than 60 pages of endnotes. The only (minor) weakness of their book is that it is so crammed full with information that it reads like 250 Nature News and Views articles back to back. The authors must have worked hard to come close, in every field, to the shortest possible description (whose size, incidentally, is one measure of complexity), but a reader relentlessly fed on such a diet of compressed information will stage a growing class of models in progress, intended to "rephrase" all instances of cas and to illustrate the emergence of complex structures through natural selection. Echo consists of populations of active elements called 'agents' that can reproduce in cyberspace. The rules are judiciously inspired from all kinds of cas, be they ecological (agents have to collect resources before they reproduce), genetical (the agents' capabilities are determined by strings), immunological (agents have tags reminiscent of epitopes), economical (agents can exchange resources) and so on. Given the right sort of coaxing, some agents ought eventually to aggregate and build hierarchical structures. The final stage should be a kind of supermodel able to mimic the embryogenesis of multicellular organisms just as well as the origins of multi-agent organizations such as industrial enter-

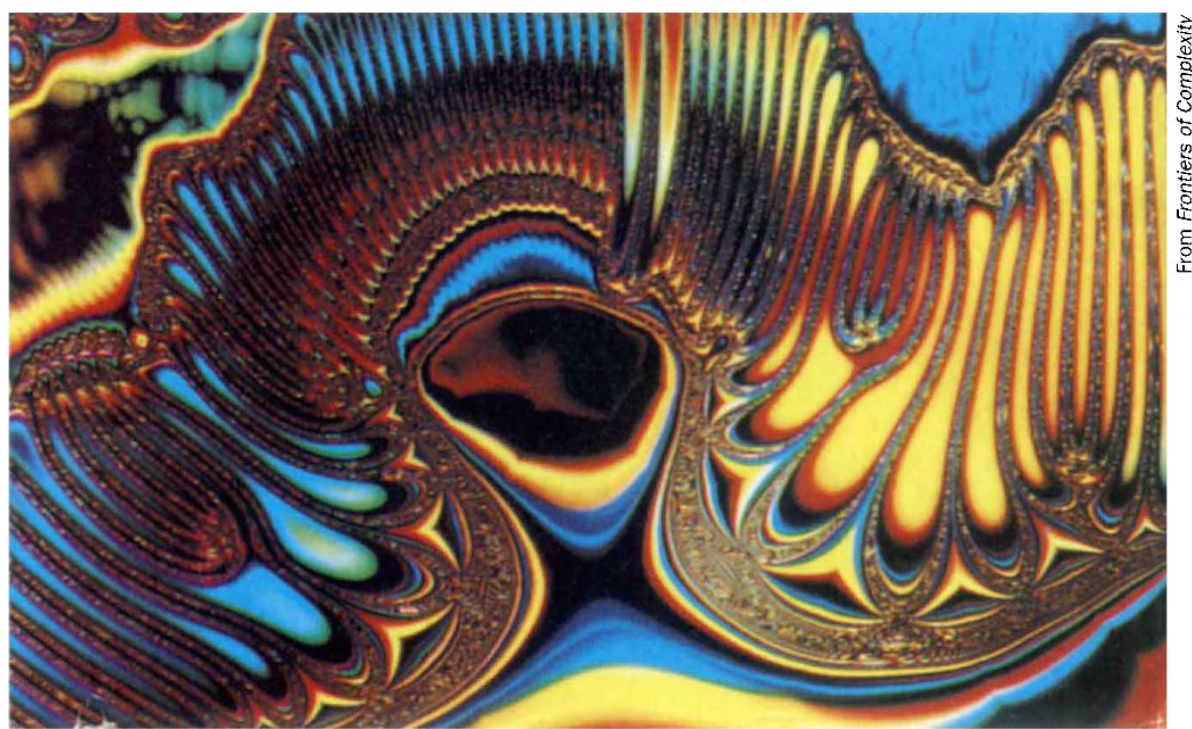

Emerging order — pattern 'bred' by Karl Sims through natural selection in a supercomputer.

occasionally long for some redundancy.

Hidden Order is much more relaxed, a casual meandering through vistas of computational ecologies. John Holland, the author, is one of the most durable figureheads of complexity. The first person to obtain a $\mathrm{PhD}$ in computer science, he has pioneered since the early 1960 s the use of genetic algorithms, where populations of computer programs evolve under mutation, selection and recombination to achieve well-adapted solutions to complex problems. Holland's approach has proved its worth and spawned a vast field of parallel problem-solving techniques that aim at rivalling classical optimization methods.

In Hidden Order, Holland describes his more recent work on cas (complex adaptive systems). He unabashedly believes that there are general principles ruling all these examples, from cell to megalopolis; warns us - rather engagingly - that "the task of formulating theory for cas is more than usually difficult"; and then proceeds to sketch the construction of Echo - ultimately the mother of all models, but at this prises. There is, Holland admits, still a long way to go.

Will this type of work ever lead to a science of complexity able to help those tackling real complex systems, for instance embryologists? Hardly so. But that is not the point. In Frontiers of Complexity we read that the young Holland used to file his ideas on adaptation in folders labelled "Glass Bead Game". This is more than just an echo of the 1960s' infatuation with Hermann Hesse's novels. It pithily resumes the role of what erroneously is termed the 'science' of complexity: it is not a science in the usual sense at all, but, like the fictitious Glass Bead Game, an intellectually supercharged exercise aimed at relating and reflecting themes from diverse disciplines, and a way of organizing the rush of new information and of gaining familiarity with emerging insights. This is why science writers adore it.

Karl Sigmund is at the Institut für Mathematik, Universität Wien, Strudlhofgasse 4, A-1090 Wien, Austria. 